\title{
Accommodation of Grain Boundary Coherency Strain by Interfacial Disconnections
}

\author{
D.L. Medlin ${ }^{1}$, D. Cohen ${ }^{1}$, R.C. Pond ${ }^{2}$, A. Serra ${ }^{3}$, J.A. Brown ${ }^{4}$, Y. Mishin ${ }^{4}$ \\ ${ }^{1}$ Sandia National Laboratories, Livermore, CA \\ ${ }^{2}$ University of Liverpool, Liverpool UK \\ ${ }^{3}$ Universitat Politècnica de Catalunya, Barcelona, Spain. \\ ${ }^{4}$ George Mason University, Fairfax VA
}

The specific mode by which strain is accommodated at a grain boundary depends ultimately on the defects that are admissible at the interface. Understanding the structure and topology of such defects is, therefore, vital to connecting atomistic and mesoscale descriptions of grain boundary behavior. In this presentation we discuss the structure and function of interfacial disconnections (i.e. line defects possessing both step and dislocation character [1]) at a $90^{\circ}<110>$ tilt boundary in gold.

An example of one such boundary, which is vicinal to $\{111\} /\{112\}$, is shown in Figure 1. Here, locally coherent $\{111\} /\{112\}$ terraces are separated by interfacial disconnections that join steps of five $\{242\}$ planes with two $\{111\}$ planes in the upper and lower crystals, respectively. We designate these defects as ""'b5/2". A strain of $5.7 \%$ is required to maintain coherency of the $\{111\}$ planes crossing the horizontal $\{112\}_{\lambda} /\{111\}_{\mu}$ terraces at this interface. We have shown that the observed array of defects efficiently accommodates this coherency strain [2]. In some cases the interfacial disconnections are dissociated. For instance, in Figure 2, a " $b_{5 / 2} "$ defect has dissociated into two components, one matching three $\{242\}$ planes with one $\{111\}$ plane $\left(b_{3 / 1}\right)$ and the other matching eight $\{242\}$ planes with three $\{111\}$ planes $\left(\mathrm{b}_{-8 /-3}\right)$. We also find this same configuration in atomistic simulations of the interface (Figure 3). By measuring the Burgers vectors of the defects, we have shown that this dissociation is favored by elastic energy criteria. Moreover, our analysis shows that the $b_{3 / 1}$ defect is glissile and is thus able move readily to the center of the $\{111\}$ terrace in response to repulsive interactions with the $b_{-8 /-3}$ defects that bound the adjacent terraces [3].

The defects we have discovered at this grain boundary are in many ways analogous to those that occur at epitaxial or tranformation interfaces between different crystallographic phases. These have a dual function in both accommodating the interfacial coherency strain and dictating the local boundary inclination. In principle, similar defects should arise at other grain boundaries. Establishing the defect properties of these and related interfacial defects provides a more fundamental understanding of the strain relief mechanisms at grain boundaries.

\section{References}

[1] J. P. Hirth, J. Phys. Chem. Solids 55, (1994) 985.

[2] D.L. Medlin, D. Cohen, R.C. Pond, Philosophical Magazine Letters 83 (4) (2003) 223.

[3] R.C. Pond, D.L. Medlin, A. Serra. Submitted to Philosophical Magazine (2006).

[4] Funding for this work was provided at Sandia by the Department of Energy, Office of Basic

Energy Science, Division of Materials Science under contract DE-AC04-94-AL85000.t 


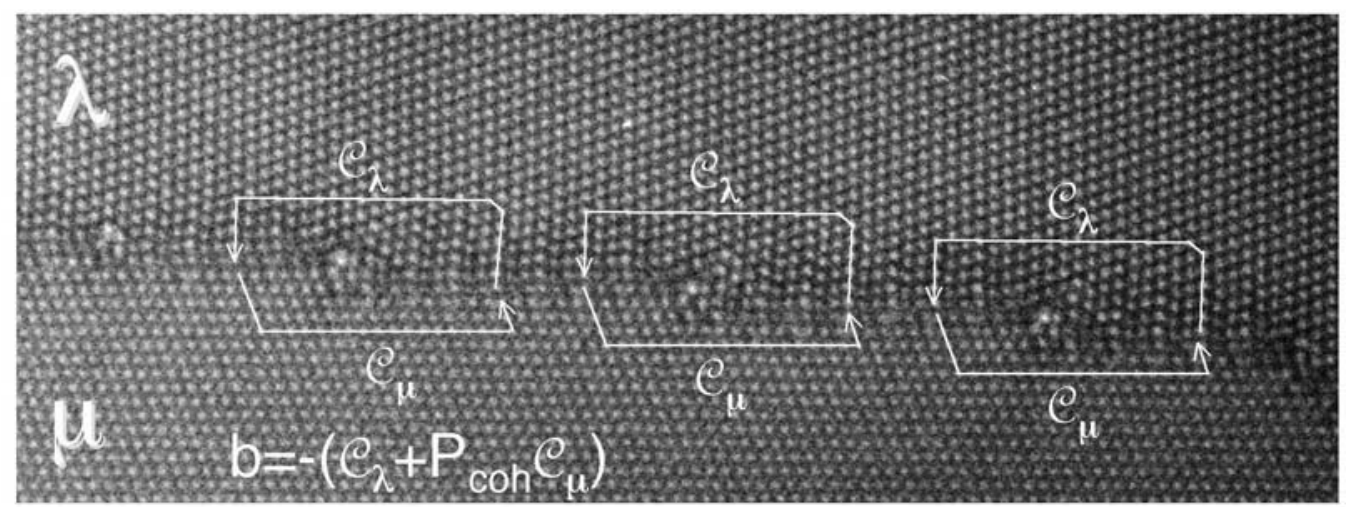

Fig. 1. HRTEM image showing interfacial disconnections between coherent $\{111\} /\{112\}$ terraces at a $90^{\circ}<110>$ boundary in $\mathrm{Au}$. The step heights correspond to 2 $\{111\}$ spacings in the lower $(\mu)$ crystal and $5\{242\}$ spacings in the upper $(\lambda)$ crystal. The Burgers vector of the disconnections is obtained from the closure failure of the two circuit paths, $C_{\mu}$ and $C_{\lambda}$, mapped into a reference frame describing the $90^{\circ}$ rotation between the two lattices and the strain required to force the two crystals into complete coherency.

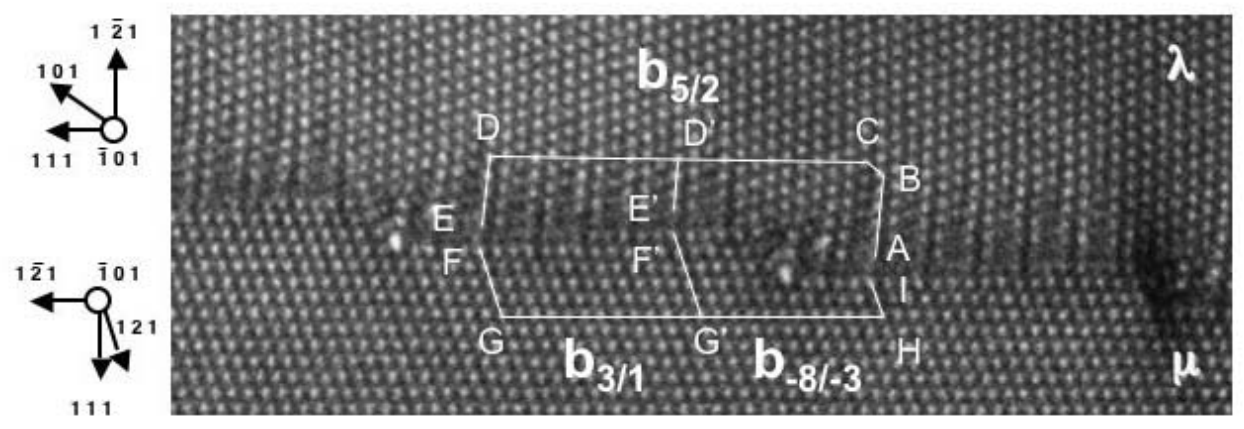

Fig. 2. Example of $b_{5 / 2}$ defect that has dissociated into two defects, $b_{3 / 1}\left(E^{\prime} E_{\lambda} F^{\prime}{ }_{\mu}\right)$ and $b_{-}$ 8/-3 $\left(\mathrm{AE}_{\lambda}^{\prime} \mathrm{F}^{\prime} \mathrm{I}_{\mu}\right)$. This analysis allows us to show that the $\mathrm{b}_{3 / 1}$ defect is glissile and that the dissociation is favored from an elastic energy criterion.

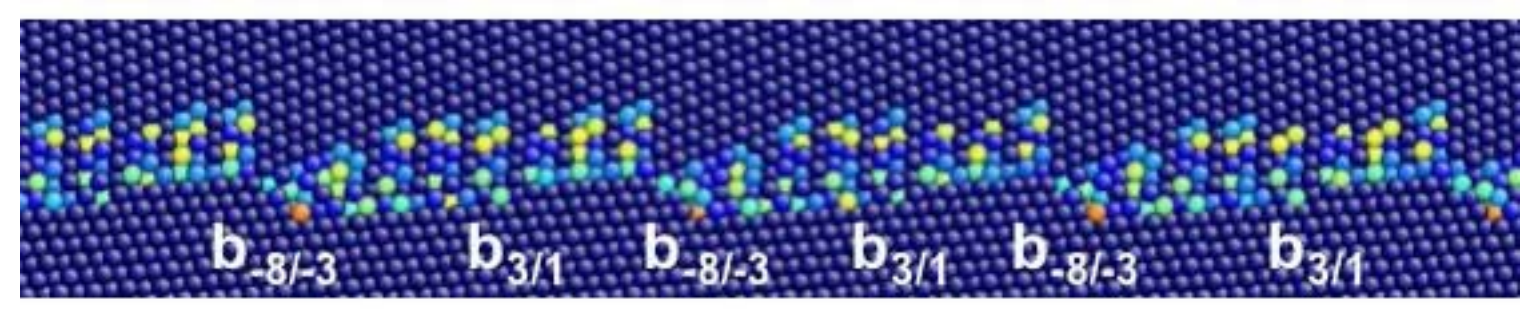

Fig. 3. Atomistic simulation (using EAM) of the $90^{\circ}<110>$ interface. The average boundary inclination in this simulation was fixed in the unrelaxed cell based on the step heights and spacing of undissociated $b_{5 / 2}$ defects required to fully accommodate the $5.7 \%$ interfacial coherency strain. Upon relaxation, these $b_{5 / 2}$ defects dissociated into an array of $b_{3 / 1}$ and $b_{-8 /-3}$ defects associated, respectively, with alternating up and down steps. 